\title{
Looking Through the Phonograph Record
}

\author{
Elements That Produce Surface Noise
}

By Francis F. Lucas

$\mathrm{S}$ - ME years ag*, when the honograph was in its earlier stages of development, vaudeville performers often entertaimed their audiences with imitations of phonographic reprouction. All of the then characteristic tinny sounds with the usual accompaniment of scratching and snapping were more or less realistically initate an the performances were quite apt to end with the repetition of a few sounds, indicative of the stylus traveling round and round in the same grove. In these lays the phonograph was more of a novelty than it was a musical instrument, but the tremendous aevel-pment which followea has mace it one of the most popular of musical instruments.

As an entertainer it probably has no equal and has been the means of bringing music and entertainment to the most remote places. During the war we rea of the sub-sea raiders lying quietly on the ocean bed while their crews listened to the latest in popular music on the phongraph. All of which gees to show that some really remarkable improvements must have been male, otherwise the demand for phonographs would have died out with the novelty. With the incentive -f polar dermand it is reasenable to expect that the devel. -pment of the future will result in more faithful reprouction and greater clarity of tone with the elimination of all extraneous noises.

Recently a laboratery study of the standar phonegraph records was undertaken with the object of comparing the different recera surfaces so that the structural elements proucing surface noise might be male more tangible and perhaps, thereby, facilitate the application of corrective measures.

Surface noise or "scratching" of phonegraph recors is one of the peculiar characteristics of honographic reprouction which, no dolut, everyone would like to see wone away with. It results from contact of stylus and recor and has several contributing causes, one of which is the character of the recer surface.

To the unaided eye the surface of a recer appears exceptionally smooth and highly polished but when played all rec- pros proce surface noise. If one were analytically inclined he probably would conclude that even though the record surface woes appear smooth and polished it must have miniature hills and ales an be more or less granular. Even with the aid of a micrescope and using metheis which are usually applied when examining opacue objects the results are not satisfactery.

For the benefit of those wh have not had actual experience in technical microscopy it may not be aniss to say that the mounting of objects for microscopic examination is somewhat of an art in itself and that the mode of procedure differs with the character of the specimen. In some cases the specimen is mounted as an opacue object and oftentimes its surface is etched with some reagent which will differentiate in the structure. In ther cases very thin an transparent sections -f the object are mounted in a suitable medium on a glass slice and protected above by a thin piece of glass called a cover glass.

For critical examination of a phongraph recor by micr scopic methods the conditions seemed to call for a very thin section from the record surface, a section of the skin so thin that it could be examined under consilerable magnification by transmitte light. In making the section the surface of the recor should not be disturied even to the slightest extent, -therwise the true conditions would not be aisclosed by the
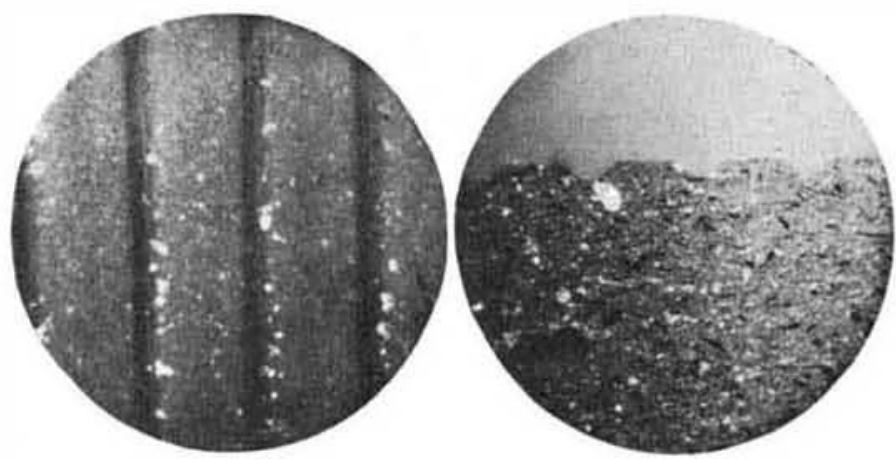

FIG. I

RECORDA 37 DIAMETERS
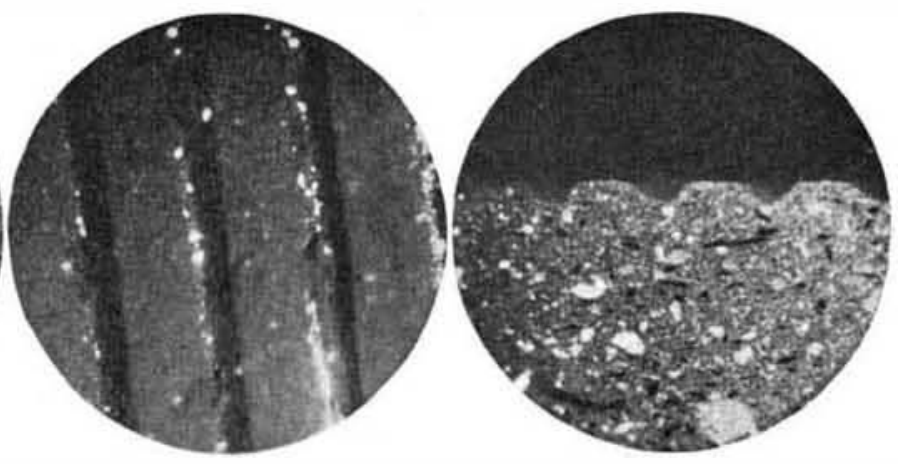

FIG. 3

RECORD B 37 DIAMETERS
FIG, 4

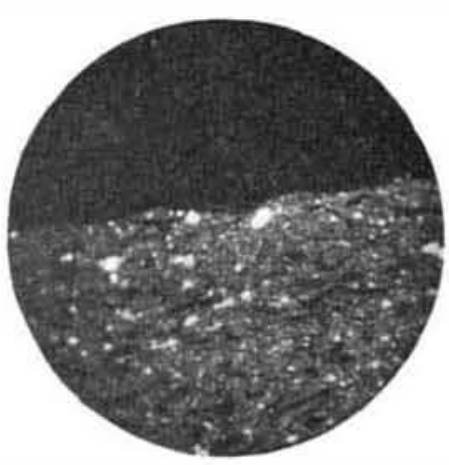

FIG. 5

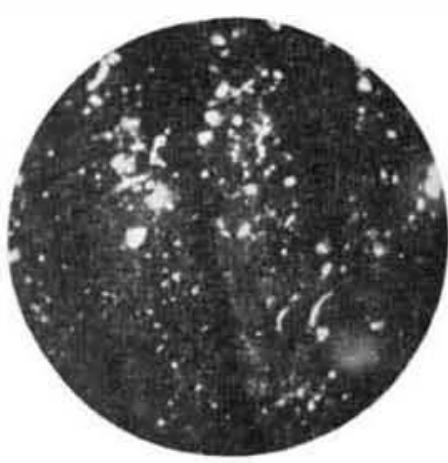

RECORD C

37 DIAMETERS
FIG. 6

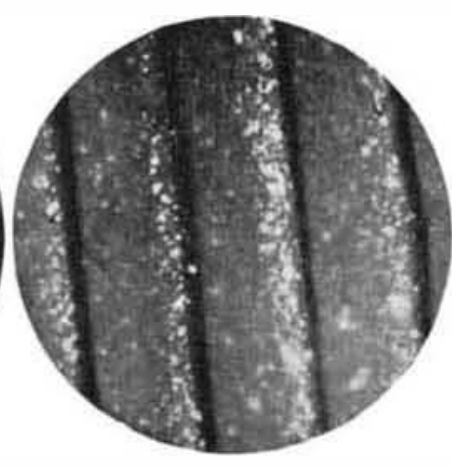

FIG. 7

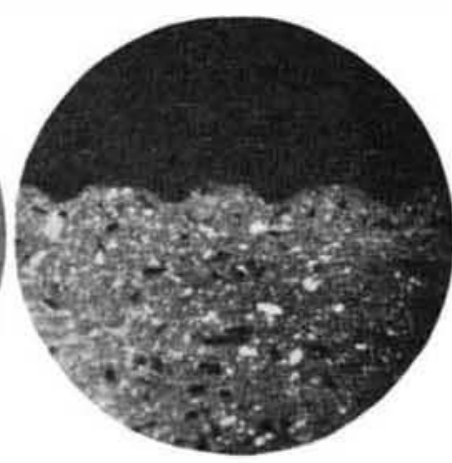

RECORD D

37 DIAMETERS
FIG. 8 

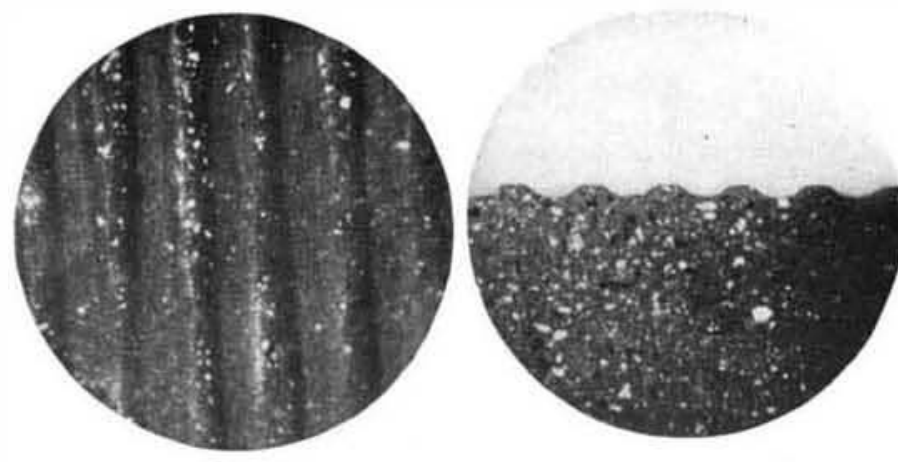

FIG. 9

RECORD E 37 DIAMETERS

FIG. 10

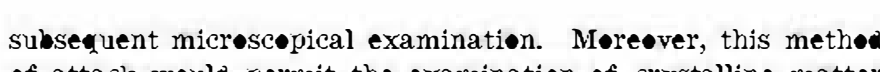
-f attack would permit the examination of crystalline matter by pelarized light and alsø, it would allow the individual particles to be gaged by micrometry.

After much laboratery work of the most painstaking ereer and innumerable failures which are usually incient to work -f this kind a delicate mode of procedure was developed where by a section of the surface measuring approximately one centimeter square and only a few thousandths of a millimeter in thickness could be removed from the record. These sections were then mounted on glass slides in the manner de scribed above after which it was possible by means of the microscope

and condition of the material forming the bottom of the recore grooves. Even to one experienced in technical micrescopy the results were startling and they seemed sufficiently convincing to warrant the speculation that the development of the phonograph recor has not as yet reached its zenith. As a further aid in the investigation transverse sections also were prepared and a metho of reproducing the record surface in a transparent medium was developed as will be described later. To reprouce the surfaces ph phomicrography proved sømew hat of a problem but by a special arrangement with a powerful illuminant together with the necessary optical sys tem the photomicrographs repruced here were taken.

In the selection of specimen recoris for examination every effort was male to secure thor ughly representative receras from each manufacturer's product. In general, unused rec-ris of the latest prouction were procured from dealers whese sales are large and careful selection it was felt that the results would be representative. In those cases where the recor manufacturer alsø markets a phon॰graph the recuras were played on a machine of the same make; otherwise they were played on one of the standar available machines arapted to the record. The recor ss selected include all of the standard makes with exception of the cheaper records in which a high degree of artistic or technical attainment should not be expected.

In order that the illustrations may be understow and cor-

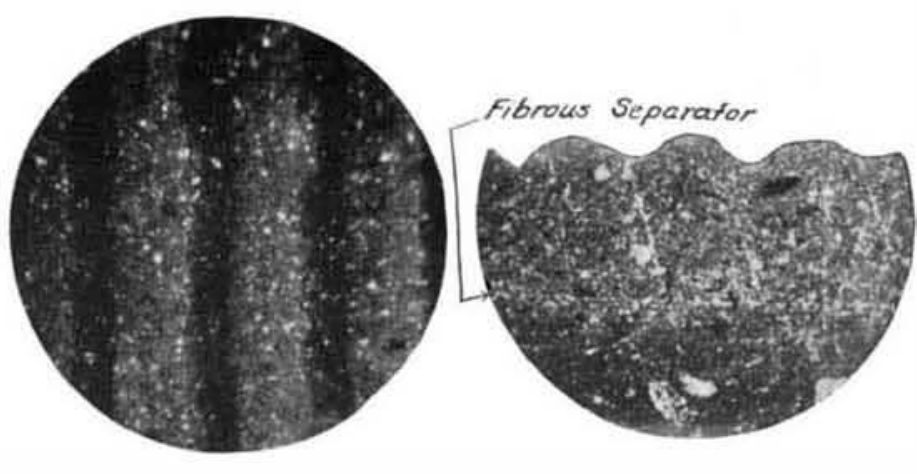

FIG. 11

RECORD F 37 DIAMETERS rectly interpreted a brief description of recor construction seems necessary. Phonograph recoris are make from that class of materials kn॰wn as hot molked composition. They consist of a binder intimately mixed with suitable mineral and vegetable fillers and a small proportion of coloring matter. Uncler suitable conditions of temperature and pressure the mixture assumes a plastic condition and may be molded. The binder may be either a natural or synthetic gum or resin, or a combination of woth. The commonly used natural resins -r gums include shellac and rosin. The synthetic resins (phenolic condensation proucts) have the property of resisting ieformation under meierately elevated temperatures and, too,

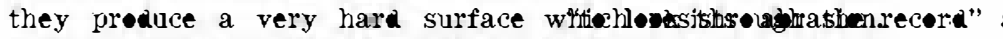
Consequently recoris made with these resins io not scratch and mar easily aring handing. The natural resinous materials are often affected by heat and records male with them are apt to deform unless properly stored. Als the surface -f such a recor is somewhat soft and apt to be damaged by careless handing. The mineral fibers, such as rottenstone, chalk, etc., are used to impart hardness and strength. The vegetable fillers are usually cotton or wood fibers and their function is to help hold the mass tenaciously together and to counteract brittleness.

Figures 1 to 19 inclusive are photomieregraphs of the nine prøminent recors which were selected for investigation. The bright spots are the particles which transmitted the light more brilliantly than the ajjacent particles and from a consiceration of the illustrations it seems evident that what to the eye appears to be smoth and polished is actually a rough and rugged road, at least in sø far as the stylus is concerned.

Fr॰m a ritical examination of the illustrations oneprobably would conclude that record " $H$ " should have the least surface noise and this assumption would check exactly with the facts. The recora consists of a lisk of cheap composition faced with a pure phenolic condensation prouct. Therefore, the recor grovves are mace up of a very smø»th, hare, homøgeneous substance, free from mineral and vegetable matter such as is found in ther records. The irregularities shown in Figure 15 were found to consist of small racks, voids and air bells and

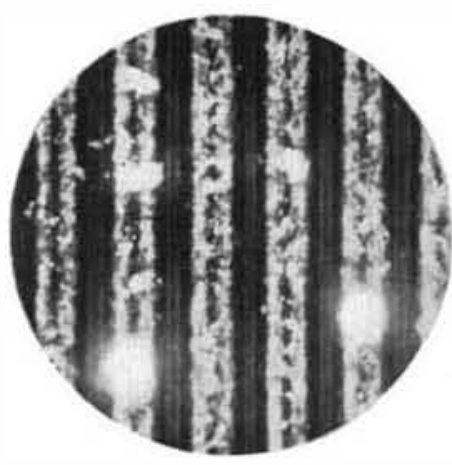

FIG. 13
RECORD G 37 DIAMETERS

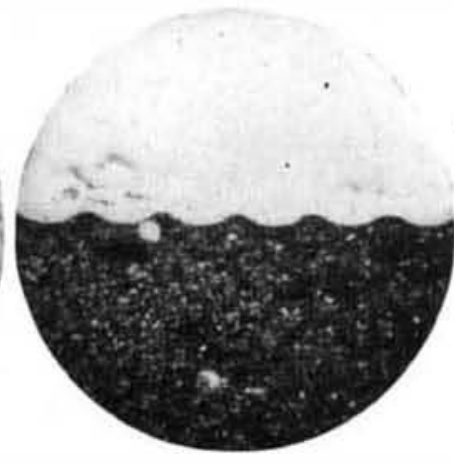

FIG. 14

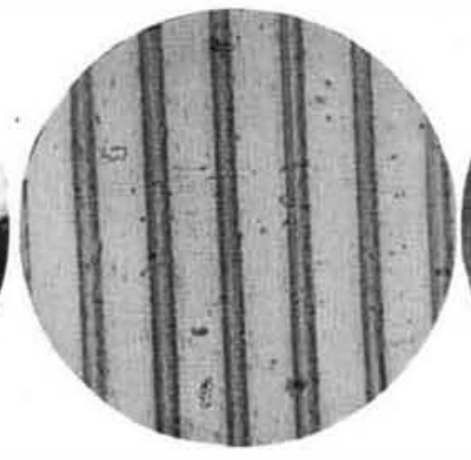

FIG. 15
RECORD $\mathrm{H}$ 37 DIAMETLRS

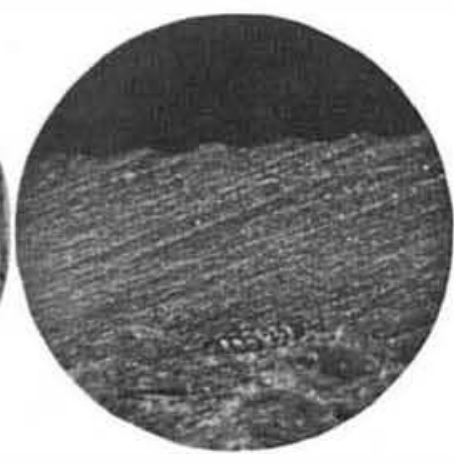

FIG. 16 

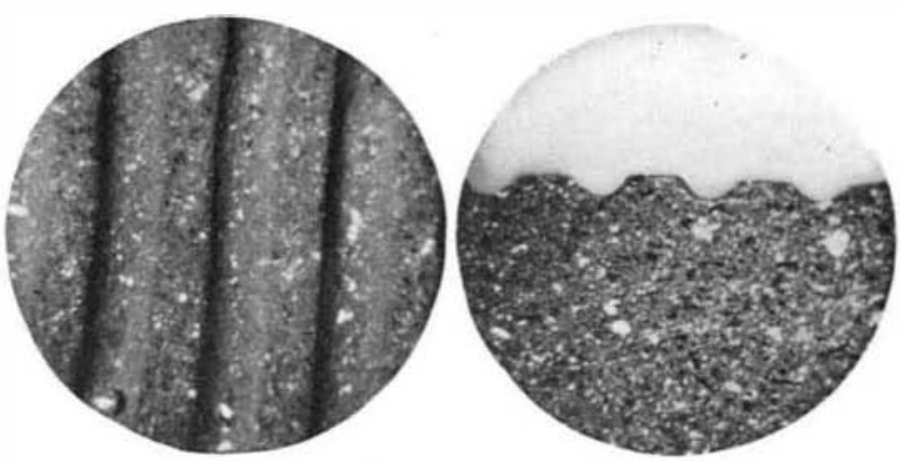

FIG. 17

RECORD I

37 DIAMETERS

FIG. 18

also of foreign particle inclusions such as dust. This photomicrograph is of special interest because it shows the order of imperfections which commercial molding may be expected to yield when working with a clear homogeneous material. So far as freedom from surface noise is concerned this record left little to be desire but unfortunately the surface coating displayed a tendency to warp and crack; due, apparently, to an inherent physical weakness in the record construction.

On the listening test record "I," Figures 17 and 18, was found to be next in order of freedom from surface noise, although there was quite a gap between it and record "H." In most of the records the surface noise was not especially pronounced and probably would not be objectionable to the average listener except, perhaps, in records reproducing soft music. Figures 17 and 18 show clean-cut molding and quite uniform structure.

In addition to the usual scratching sound, occasional "snaps and cracks" are often heard and these seem to have their origin in a different source. In record "B," Figure 3, certain imperfections in the record surface are to be seen and it seems probable that when the stylus strikes such obstructions as these the reproducer must register some sort of a violent protest. These molding imperfections were found to be more pronounced in this particular record, but they were by no means absent in the other records. Record "B" also had considerable surface noise which one would expect from the nature of the structure as shown by Figures 3 and 4 .

Record "F," Figures 11 and 12, illustrates a novelty in construction. In Figure 11 it will be seen that the record body consists of a coarse structure which is faced with a finer composition. Between the body and the surface coating on each side is a fibrous separator. The surface of this record is of finer structure than record " $B$ " and, as might be expected, the surface noise was less intense.

Records "A," "C," "D" and "E" on the listening test were found to be much the same so far as surface noise was concerned. Some differences did exist but it will be seen from the illustrations that the surfaces are inclined to be rough and in some instances the structure is coarse.

As might be expected from Figures 13 and 14 record "G" had the most surface noise of any of the records studied. In some rec. ords the scratching assumed very disagreeable proportions and detracted greatly from the musical value of the record. The grooves were found to consist of SPRUCE SEPARATED BY CHEM ICAL MEANS. ENILARGED 37 DIAMETERS
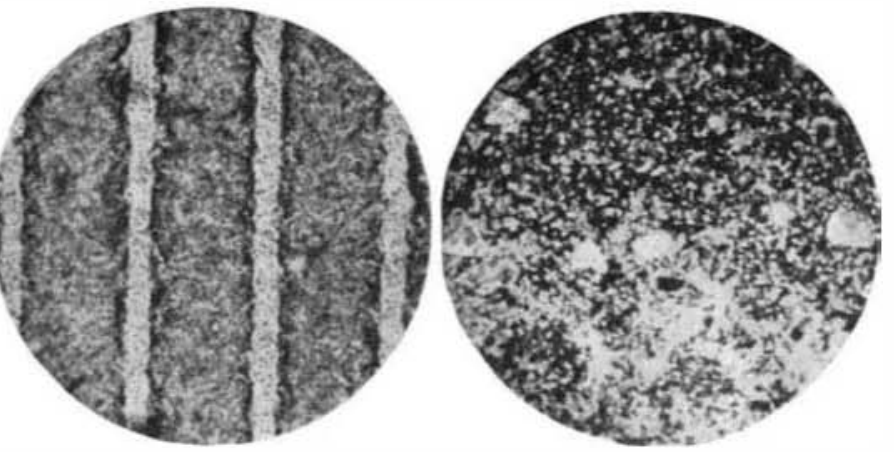

TRANSPARENCY 75 DIAN. FIG. 19

RECORD G

37 DIAIM

FIG. 20

small and large mineral particles and splintery bundles of wood fiber. The large mineral particles were present in abundance as will be noted and their origin was a mystery until treated by a method of microscopic analysis. Since a large proportion of the record consisted of wood flour it was decided to examine some representative samples of this material. The results showed that the wood flour carried large inclusions of mineral particles similar to those found in the record surface. Following the woed flour to its origin it was found to consist of sawdust ground to fineness in a stone mill. Apparently in this way particles of the grindstones were being conveyed to the record surface. In Figure 22 the coarse and splintery structure of the wood flour is shown by means of a photomicrograph taken by dark ground illumination. A large mineral particle is much in evidence. An idea of the extent to which mineral particles were found to be present in wood flour may be judged from Figure 20 which shows the inorganic matter found in about one per cent of the wood flour which could be heaped on a ten-cent piece. The wisdom of using material of this kind would seem open to serious consideration, especially in view of the fact that chemically separated wood fiber free from foreign inclusions may readily be obtained. Wood fiber of this nature is illustrated in Figure 21. It will be noted that the wood has been reduce to its ultimate fiber, which is the best possible condition for molding plastics.

As a further aid in the investigation a transparent medium which would take an impression from the record surface was developed, the idea being that differences in level would be disclosed by differences in the depth of color of the impression when viewe by transmitted light. That is, a mount of this kind when examined under the miscroscope would constitute a relief map of the record surface. Figure $\mathbf{1 9}$ is from an impression taken from record "G" and the similarity of Figures 13 and 19 is quite evident. In Figure 19 the light colored bands are the ridges and the broad darker bands are the grooves from which it is clear that a dark area indicates a

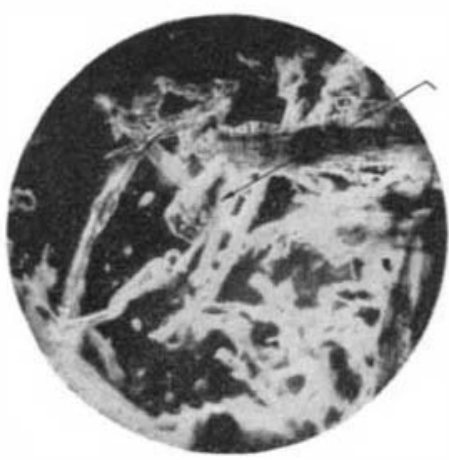

FIG. 22. GROUND WOOD FLOUR WITH COARSE MINERAL PAR TICLE INDICATED BY THE ARROW depression in the record surface and a light area an elevation. This method of examination has the advantage of being quicker but does not permit qualitative and quantitative analyses by means of the microscope.

From a consideration of the data obtained it would seem that future improvements in surface conditions appear most hopeful along lines which will result in a finer and a more homogeneous structure. 